Introduction Prevalence and incidence of HIV infection and other sexually transmitted infections (STIs) are particularly high among adolescent girls in sub-Saharan Africa. The role played by the vaginal microbiota in susceptibility to HIV and other STIs in adolescent girls is unclear. The aim of this study was to characterise the vaginal microbiota of adolescent girls in Tanzania around the time of their sexual debut.

Methods Girls aged 17-18 years old attending secondary schools in Mwanza City were invited to join a cross-sectional study. After informed consent/assent, girls were interviewed and vaginal swabs were obtained and tested for the following species by inhouse quantitative PCR: Lactobacillus crispatus, L. iners, L. gasseri, L. jensenii, L. vaginalis, Atopobium vaginae, and Gardnerella vaginalis. Differences in the prevalence of bacterial species were analysed using logistic regression.

Results Of the 403 girls enrolled, 385 provided samples for this analysis. Of these, 163 reported having had sexual intercourse. Prevalences of bacterial species were as follows (overall; sexually active/sexually naïve): L. crispatus $(69 \% ; 60 \% / 75 \%)$, L. iners (83\%; 85\%/80\%), L. gasseri $(22 \% ; 21 \% / 23 \%)$, L. jensenii (49\%; $40 \% / 55 \%)$, L. vaginalis $(66 \% ; 55 \% / 74 \%)$, A. vaginae $(44 \%$; $56 \% / 34 \%)$, and G. vaginalis (62\%; 75\%/52\%). Prevalences of A. vaginae and $G$. vaginalis were higher among girls who reported sexual intercourse (OR: 2.5; 95\% CI: 1.7-3.8 and OR: 2.8; 95\% CI: 1.8-4.4, respectively), while prevalences of L. crispatus, $\mathrm{L}$. jensenii, and $\mathrm{L}$. vaginalis were lower in sexually active girls (OR: 0.5 ; 95\% CI: $0.3-0.8$, OR: 0.5 ; 95\% CI: $0.4-0.8$, and OR: 0.4 ; 95\% CI: 0.3-0.6, respectively).

Conclusion Among girls attending secondary school in Tanzania, sexual debut was associated with quantifiable changes in vaginal microbiota. BV-associated bacteria were present in many girls before reported sexual debut. Additionally, the prevalence of L. crispatus was higher than expected; a recent study showed the prevalence among African women to be relatively low (17$38 \%)$. This challenges the view that women in sub-Saharan Africa have less L. crispatus colonisation.

Disclosure of interest statement The authors do not have a conflict of interest. No pharmaceutical grants were received in the development of this study.

\section{P03.15 PEER EDUCATION IN PRACTICE: AN EXAMPLE OF A YOUTH-LED SEXUAL HEALTH PROGRAM}

R Fuller ${ }^{*}, \mathrm{~K}$ Carter. Youth Affairs Council of Western Australia

\subsection{6/sextrans-2015-052270.243}

Introduction It's no secret that young people seek sexual health information from other young people, and peer education is an effective health promotion strategy to engage with young people about sexual health. The Youth Affairs Council of Western Australia (YACWA)'s 'Youth Educating Peers' (YEP) Project is a youth-led peer education program, which aims to give young people opportunities to connect with other young people, and take action in regards to sexual health and blood-borne virus (BBV) issues.

Methods After extensive research on peer-led sexual health education in the WA youth sector, YACWA established the YEP Crew, a group of volunteer peer educators. These young people aim to reduce the rates of sexually transmitted infections (STIs) and BBVs in WA youth by building young peoples' capacity to negotiate safer sexual practices. The YEP Crew meet regularly to design and deliver sexual health interventions through online social media, activity based workshops, and outreach at various youth sector events.

Results The YEP Crew currently has 17 active peer educators. Since July 2014 to present, the YEP Crew have facilitated seven education workshops, attended three opportunistic testing events, and conducted outreach at four festival and community events. Online, YEP Crew have a following of over 620 people on Facebook with a peak post reach of over 1,500. Young people who participate in YEP Crew workshops report increases in their knowledge of sexual health and BBV issues as well as feeling more 'confident' and 'prepared'.

Conclusion In an environment where STI rates in young people are rapidly increasing, peer education models are an effective strategy to provide relevant and engaging sexual health information focusing on protective behaviours. This model of engagement allows us to keep on top of regularly changing sexual health issues for young people, which can inform policy development and guide research priorities.

Disclosure of interest statement Nothing to declare.

\section{P03.16 SEXUAL ABUSE REPORTED BY YOUNG AUSTRALIAN WOMEN}

1,2,35M Garland ${ }^{*}$, 1,3,4Y Jayasinghe, ${ }^{5,6}$ ET Callegari, ${ }^{7}$ A Gorelik, ${ }^{1,2}$ AK Subasinghe, $5,6 \mathrm{JD}$ Wark, on behalf of the YFH, Safe-D study groups. ${ }^{1}$ Department of Microbiology and Infectious Diseases, The Royal Women's Hospital, Parkville, VIC, Australia; ${ }^{2}$ Murdoch Childrens Research Institute, Parkville, VIC, Australia; ${ }^{3}$ Department of Obstetrics and Gynaecology, University of Melbourne, Parkville, VIC, Australia; ${ }^{4}$ Department of Gynaecology, Royal Children's Hospital, Parkville, VIC, Australia; ${ }^{5}$ Royal Melbourne Hospital Department of Medicine, University of Melbourne, Parkville, VIC, Australia; ${ }^{6}$ Bone and Mineral Medicine, Royal Melbourne Hospital, Parkville, VIC, Australia; 'Melbourne EpiCentre, Royal Melbourne Hospital, University of Melbourne, Parkville, VIC, Australia

\subsection{6/sextrans-2015-052270.244}

Introduction Young women are under-represented in health research despite their transitioning a life stage critical for future health outcomes. Sexual abuse has become a critical issue, globally, leading to significant long term morbidity. We investigated the prevalence of unwanted sexual experiences (USEs) in young women, residing in Victoria, Australia.

Methods The Young Female Health Initiative (YFHI) and Safe-D are comprehensive studies of physical, sexual, reproductive, and mental health of young females. Participants (aged 16-25 years) are recruited through Facebook. Consenting participants complete an extensive online health survey and attend a site visit. USEs are assessed using questionnaires and a protocol for protection of participants developed to ensure their safety.

Results Data available for 398 participants (YFHI: $\mathrm{N}=178$; Safe-D: $N=220$ ) shows $14 \%$ recalled a USE when they were < 16 years of age, of whom, 62\% indicated they suffered from depression, 32\% reported penile-genital contact, and 20\% had been diagnosed by a doctor or health professional with a sexually transmitted infection. In women who recalled a USE between $16-18$ years of age $(60 / 398,15 \%), 42 \%$ stated they felt pressured into sexual intercourse with a man, and $28 \%$ reported having been raped. In women aged $\geq 18$ years, no significant differences were observed between the proportion of women from the YFHI study, who reported a USE at $<16$ years of age (19\%), compared to participants from the Safe-D study (11\%, $\mathrm{p}>0.05)$.

Conclusion To our knowledge this is the first Australian study in which the prevalence of USE in women as young as 16 years has been assessed. Prevalence was lesser than in other Australian 